\title{
Shape equations for axisymmetric vesicles: A clarification
}

\author{
Frank Jülicher and Udo Seifert \\ Institut für Festkörperforschung, Forschungszentrum Jülich, 52425 Jülich, Germany
}

(Received 25 June 1993)

\begin{abstract}
We derive the shape equations for axisymmetric vesicles and show that they are identical to the general shape equation [Ou-Yang Zhong-Can and W. Helfrich, Phys. Rev. A 39, 5280 (1989)] specialized to axisymmetry. We consider three different topologies (an axisymmetric membrane segment suspended between two circular rings and closed vesicles of spherical and toroidal topology). We point out that the shape equations are independent of the variational method used.
\end{abstract}

PACS number(s): 82.70.-y

\section{INTRODUCTION}

Vesicles are closed surfaces formed by lipid bilayers in aqueous solution. Their shape is determined by the minimum of the bending energy of the membrane subject to constraints on the total area and the enclosed volume (for reviews, see [1-3]). In the so-called spontaneous curvature model, the bending energy is given by $[4,5]$

$$
F \equiv \frac{\kappa}{2} \oint d A\left(2 H-C_{0}\right)^{2},
$$

where $\kappa$ is the bending rigidity. The mean curvature on the surface is denoted by $H$ while $C_{0}$ is the spontaneous curvature. The volume and area constraints can be incorporated in the energy minimization by introducing the Lagrange multipliers $P$ and $\Sigma$ which play the roles of a pressure and a tension. Minima of the bending energy are then stationary shapes of the functional $F^{\prime}=F+\Sigma A+P V$.

The variation of the functional $F^{\prime}$ with respect to arbitrary normal displacements has been performed by $\mathrm{Ou}$ Yang and Helfrich $[6,7]$. It leads to the general shape equation

$\bar{P}+2 \bar{\Sigma} H-\left(2 H-C_{0}\right)\left(2 H^{2}-2 K+C_{0} H\right)-2 \Delta_{b} H=0$

where $\bar{P}=P / \kappa$ and $\bar{\Sigma}=\Sigma / \kappa . \Delta_{b}$ is the Laplacian on a curved surface and $K$ denotes the Gaussian curvature on the surface.

Numerically, a large class of axisymmetric vesicles of spherical and toroidal topology has been calculated as stationary points of the functional $F^{\prime}$ [5,8-12]. For these calculations, special shape equations have been derived assuming axisymmetry from the beginning. Recently, it has been claimed [13] that the shape equations which have been used in these studies differ from the general shape equation (2). Thus, doubts have been cast on the results obtained in these works. The purpose of this paper is to show explicitly that the shape equations which have been used in these studies are correct and compatible with the general shape equation. Thus, previous numerical results are correct.
This paper is organized as follows. In Sec. II, we derive the first variation of $F^{\prime}$ for axisymmetric membranes. This variation comprises a bulk part and boundary terms. It turns out that the vanishing of the bulk part leads to the same equation as if the general shape equation (2) is specialized to axisymmetry, provided the boundary terms are treated properly. In Sec. III, we study different topologies and show that the shape equations for the case of vesicles of spherical topology simplify. In Sec. IV, we point out the errors made in Ref. [13].

\section{SHAPE EQUATION FOR AXISYMMETRIC MEMBRANES}

An axisymmetric surface can be parametrized by the angle $\psi(S)$, where $S$ is the arclength along the contour [8] (see Fig. 1). The coordinates $R(S)$ and $Z(S)$ depend on $\psi(S)$ through

$$
\dot{R}=\cos \psi \quad \text { and } \quad \dot{Z}=-\sin \psi,
$$

where the dot denotes the derivative with respect to the arclength $S$.
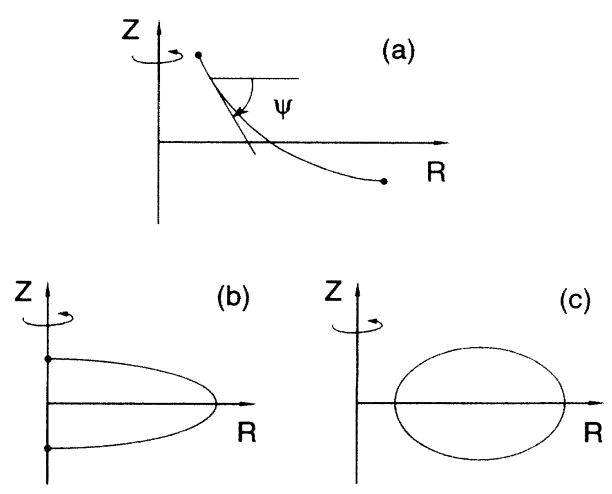

FIG. 1. Three different topologies for an axisymmetric membrane configuration: (a) membrane suspended between two rings, (b) vesicle of spherical topology, and (c) vesicle of toroidal topology. The contour is parametrized by $R, Z$, and the tilt angle $\psi$ as a function of the contour length $S$. 
The functional to be minimized can be written as

$$
F^{\prime} \equiv 2 \pi \kappa \int_{S_{1}}^{S_{2}} d S L
$$

with

$$
\begin{aligned}
L \equiv & \frac{R}{2}\left(\dot{\psi}+\frac{\sin \psi}{R}-C_{0}\right)^{2}+\bar{\Sigma} R+\frac{\bar{P}}{2} R^{2} \sin \psi \\
& +\gamma(\dot{R}-\cos \psi)+\eta(\dot{Z}+\sin \psi),
\end{aligned}
$$

using

$$
2 H=\dot{\psi}+\sin \psi / R \text {. }
$$

The Lagrange multiplier functions $\gamma(S)$ and $\eta(S)$ have to be introduced to guarantee the geometrical relations (3). With the variation

$$
\begin{aligned}
\psi(S) & =\psi_{0}(S)+\delta \psi(S) \\
R(S) & =R_{0}(S)+\delta R(S) \\
Z(S) & =Z_{0}(S)+\delta Z(S) \\
\gamma(S) & =\gamma_{0}(S)+\delta \gamma(S) \\
\eta(S) & =\eta_{0}(S)+\delta \eta(S) \\
S_{1} & =S_{1,0}+\delta S_{1} \\
S_{2} & =S_{2,0}+\delta S_{2}
\end{aligned}
$$

one finds for the variation of the functional $F^{\prime}$ the expression

$$
\begin{aligned}
\delta F^{\prime}= & \int_{S_{1}}^{S_{2}} d S\left\{\left[\frac{\partial L}{\partial \psi}-\frac{d}{d S} \frac{\partial L}{\partial \dot{\psi}}\right] \delta \psi+\left[\frac{\partial L}{\partial R}-\frac{d}{d S} \frac{\partial L}{\partial \dot{R}}\right] \delta R\right. \\
& \left.+\left[\frac{\partial L}{\partial Z}-\frac{d}{d S} \frac{\partial L}{\partial \dot{Z}}\right] \delta Z+\frac{\partial L}{\partial \gamma} \delta \gamma+\frac{\partial L}{\partial \eta} \delta \eta\right\} \\
& -\left.\mathcal{H} \delta S\right|_{S_{1}} ^{S_{2}}+\left.\frac{\partial L}{\partial \dot{\psi}} \delta \psi\right|_{S_{1}} ^{S_{2}}+\left.\frac{\partial L}{\partial \dot{R}} \delta R\right|_{S_{1}} ^{S_{2}}+\left.\frac{\partial L}{\partial \dot{Z}} \delta Z\right|_{S_{1}} ^{S_{2}}
\end{aligned}
$$

Here, $\mathcal{H} \equiv-L+\dot{\psi} \partial L / \partial \dot{\psi}+\dot{R} \partial L / \partial \dot{R}+\dot{Z} \partial L / \partial \dot{Z}$ is the Hamiltonian function and $\partial L / \partial \dot{\psi}=R\left(2 H-C_{0}\right)$, $\partial L / \partial \dot{R}=\gamma, \partial L / \partial \dot{Z}=\eta$. The bulk part vanishes for arbitrary variations if the usual Euler-Lagrange equations are satisfied, which are given by Eqs. (3) and

$$
\begin{aligned}
\ddot{\psi}= & \frac{\cos \psi \sin \psi}{R^{2}}-\frac{\dot{\psi}}{R} \cos \psi+\frac{\bar{P}}{2} R \cos \psi \\
& +\frac{\gamma}{R} \sin \psi+\frac{\eta}{R} \cos \psi, \\
\dot{\gamma}= & \frac{1}{2}\left(\dot{\psi}-C_{0}\right)^{2}-\frac{\sin ^{2} \psi}{2 R^{2}}+\bar{\Sigma}+\bar{P} R \sin \psi, \\
\dot{\eta}= & 0 .
\end{aligned}
$$

Since the variation of $F^{\prime}$ with respect to a variation of the contour lengths $\delta S_{1}$ and $\delta S_{2}$ at the two end points has to vanish, one obtains the condition $\mathcal{H}\left(S_{1}\right)=\mathcal{H}\left(S_{2}\right)=0$. Moreover, $\mathcal{H}$ is conserved along the contour because $L$ does not depend on the arclength $S$. We thus get the additional equation

$$
\begin{aligned}
\mathcal{H}= & \frac{R}{2}\left[\dot{\psi}^{2}-\left(\frac{\sin \psi}{R}-C_{0}\right)^{2}\right]-\frac{\bar{P}}{2} R^{2} \sin \psi-\bar{\Sigma} R \\
& +\gamma \cos \psi-\eta \sin \psi=0
\end{aligned}
$$

for a stationary contour.

To compare these equations with the general shape equation (2), we transform them into a form without Lagrange multipliers $\gamma$ and $\eta$. First Eq. (12) is rewritten as $\eta=\eta(\psi, \dot{\psi}, R, \gamma)$. This expression for $\eta$ is now inserted into Eq. (9) which afterwards is rewritten as $\gamma=\gamma(\psi, \dot{\psi}, \ddot{\psi}, R)$. To eliminate $\gamma, d \gamma / d S$ obtained from this equation is inserted as $\dot{\gamma}$ into Eq. (10). After reparametrization $[\psi(S), R(S)] \rightarrow \psi(R)$ one obtains

$$
\begin{aligned}
\psi^{\prime \prime \prime} \cos ^{3} \psi= & 4 \sin \psi \cos ^{2} \psi \psi^{\prime \prime} \psi^{\prime}-\cos \psi\left[\sin ^{2} \psi-\left(\cos ^{2} \psi\right) / 2\right] \psi^{3}+\frac{7 \sin \psi \cos ^{2} \psi}{2 R} \psi^{2}-\frac{2 \cos ^{3} \psi}{R} \psi^{\prime \prime} \\
& +\left[\frac{C_{0}^{2}}{2}-\frac{2 C_{0} \sin \psi}{R}+\frac{\sin ^{2} \psi}{2 R^{2}}+\bar{\Sigma}-\frac{\sin ^{2} \psi-\cos ^{2} \psi}{R^{2}}\right] \cos \psi \psi^{\prime} \\
& +\left[\bar{P}+\frac{\bar{\Sigma} \sin \psi}{R}-\frac{\sin ^{3} \psi}{2 R^{3}}+\frac{C_{0}^{2} \sin \psi}{2 R}-\frac{\sin \psi \cos ^{2} \psi}{R^{3}}\right]
\end{aligned}
$$

where the prine denotes a derivative with respect to $R$. Precisely the same equation is obtained if the general shape equation (2) is specialized to the axisymmetric case parametrized by $\psi(R)$. The latter calculation has been performed by $\mathrm{Hu}$ Jian-Guo and Ou-Yang ZhongCan $[13,14]$.

Thus, we have shown explicitly that the shape equation for an axisymmetric membrane and the general shape equation specialized to an axisymmetric membrane are identical. Note that it was crucial to allow explicitly for variations in the contour length, which leads to Eq. (12).
Note also that we have not yet imposed that the last three boundary terms in (8) vanish.

\section{SHAPE EQUATIONS IN DIFFERENT TOPOLOGIES}

\section{A. Axisymmetric membrane segment}

Consider the somewhat artificial conformation of an axisymmetric membrane segment suspended at two rings 
[see Fig. 1(a)]. In this geometry, $\delta Z$ and $\delta R$ have to vanish at both boundaries. Therefore, the only remaining boundary term in (8) is the one involving $\delta \psi$. It vanishes if $2 H\left(S_{1}\right)=2 H\left(S_{2}\right)=C_{0}$. Note that in general the value of the parameter $\eta$ and the boundary values $\gamma\left(S_{1}\right)$ and $\gamma\left(S_{2}\right)$ of $\gamma(S)$ are nonzero for such a conformation.

\section{B. Vesicles of spherical topology}

For a vesicle of spherical topology, $S=S_{1}$ corresponds to the north pole and $S=S_{2}$ corresponds to the south pole [compare Fig. 1(b)]. Therefore, the variations $\left.\delta Z\right|_{S=S_{1}}$ and $\left.\delta Z\right|_{S=S_{2}}$ are independent. This leads immediately to $\eta=0$. The remaining two boundary terms in (8) vanish because $\psi$ and $R$ are fixed at the poles. Assuming spherical topology from the beginning, Eqs. (9-11) and (12) with $\eta=0$ have been derived in Ref. [8].

It is instructive to eliminate the Lagrange multiplier function $\gamma(S)$ for the case of spherical topology directly. To this end, one rewrites Eq. (9) with $\eta=0$ as $\gamma=$ $\gamma(\psi, \dot{\psi}, \ddot{\psi}, R)$. Inserting this expression for $\gamma$ into Eq. (12) results in another differential equation for $\psi(S)$. After a parameter change $[\psi(S), R(S)] \rightarrow \psi(R)$, one obtains a second order differential equation

$$
\begin{aligned}
\psi^{\prime \prime} \cos ^{2} \psi= & \frac{\sin \psi \cos \psi}{2} \psi^{\prime 2}-\frac{\cos ^{2} \psi}{R} \psi^{\prime} \\
& +\frac{\sin 2 \psi}{2 R^{2}}+\frac{\bar{P} R}{2 \cos \psi}+\frac{\bar{\Sigma} \sin \psi}{\cos \psi} \\
& +\frac{\sin \psi}{2 \cos \psi}\left(\frac{\sin \psi}{R}-C_{0}\right)^{2}
\end{aligned}
$$

This equation is also obtained if the contour is directly parametrized by $\psi(R)$ without Lagrange multipliers $\gamma$ and $\eta[13]$.

Note that for spherical topology, the equation for $\psi(R)$ as given by Eq. (14) is of second order whereas in the general case restricted to axisymmetry Eq. (13) it is of third order. It should be obvious that Eq. (14) only applies to vesicles of spherical are topology because only for this topology are the variations of $\delta Z$ at the two end points independent, which leads to $\eta=0$. Solutions to Eq. (14) will also solve Eq. (13) but not vice versa.

\section{Vesicles of toroidal topology}

For vesicles of toroidal topology, the variations at the boundary are not independent since $\left.\delta \psi\right|_{S=S_{1}}=\left.\delta \psi\right|_{S=S_{2}}$, $\left.\delta R\right|_{S=S_{1}}=\left.\delta R\right|_{S=S_{2}}$ and $\left.\delta Z\right|_{S=S_{1}}=\left.\delta Z\right|_{S=S_{2}}$ [see Fig. 1(c)]. Consequently, the three boundary terms in Eq. (8) vanish without imposing any conditions on $\eta, \gamma\left(S_{1}\right)$, and $\gamma\left(S_{2}\right)$. Especially, one has to leave $\eta \neq 0$ in the shape equations. In fact, the set (9-12) has been used in Refs. $[11,12]$ to determine axisymmetric shapes of toroidal topology.

\section{DISCUSSION}

We now discuss these results in relation to work by $\mathrm{Hu}$ Jian-Guo and Ou-Yang Zhong-Can [13] who compare four different methods to obtain shape equations: (a) they specialize the general equation (2) to the axisymmetric case which leads to Eq. (13); (b) they parametrize the contour with $R(S)$ and $\psi(S)$ as a function of the contour length $S$; (c) they parametrize the contour by $\psi(R)$; and (d) they vary the axisymmetric functional with respect to a normal displacement. They find that the shape equations obtained by the methods (a)-(c) are different. In particular, methods (a)-(c) lead to different relations between the parameters $\bar{\Sigma}, \bar{P}$, and $C_{0}$ for cylinders as well as for the Clifford torus [15]. They conclude that the results of the variation depend on the choice of the parametrization and claim that the shape equations derived by methods (a) and (d) are correct while those obtained by methods (b) and (c) are wrong.

We now point out how inconsistencies arise from an improper treatment of the variations at the boundaries in methods (b) and (c). The way the shape equations are derived by methods (b) and (c) in Ref. [13] implicitly assumes that the variations of $\delta Z$ at both boundaries are independent because only then can the term $\eta(\dot{Z}+$ $\sin \psi$ ) in the Lagrangian function be omitted as is done in method (b) [see Eqs. (9) and (13) in Ref. [13]]. Likewise, one would have to include a similar term in method (c) if there were any condition on $Z(0)$ or $Z(S)$. Thus, the shape equations derived in Ref. [13] by methods (b) and (c) are only suitable for contours of vesicles of spherical topology, because only in this case are the variations of $\delta Z$ at both ends independent. Consequently they both lead to the correct condition

$$
\bar{P} R_{1}^{3}+2 \bar{\Sigma} R_{1}^{2}+C_{0}^{2} R_{1}^{2}-2 C_{0} R_{1}=0
$$

for a sphere as observed in Ref. [13]. However, one should clearly not use these equations to determine the condition under which a Clifford torus becomes a stationary shape of $F^{\prime}$.

By deriving the shape equations for a contour parametrized by the arclength $S$ [method (b)], an additional problem arises. The authors of Ref. [13] overlooked the variation of $F^{\prime}$ with respect to the total contour length which leads to the additional condition (12). Their third order shape equation for method (b) (see Eq. (13) in Ref. [13]) includes implicitly an additional constraint of fixed total contour length which in general leads to unphysical results with $\mathcal{H} \neq 0$. In the case of spherical topology such unphysical solutions are singular at the poles [16]. However, this mistake does not show up if a sphere, for which $\mathcal{H}=0$, is inserted into their shape equation for method (b). The subspace of solutions with $\mathcal{H}=0$ of this equation is identical to the solutions of the correct second order shape equation (14) for vesicles of spherical topology.

For a cylinder with radius $R_{1}$, method (b) leads to

$$
2 \bar{P} R_{1}^{3}+2 \bar{\Sigma} R_{1}^{2}+C_{0}^{2} R_{1}^{2}-1=0
$$


while method (c) leads to

$$
\bar{P} R_{1}^{3}+2 \bar{\Sigma} R_{1}^{2}+\left(C_{0} R_{1}-1\right)^{2}=0 .
$$

Equation (16) describes stationarity with respect to radius variations and is thus obtained with methods (a) and (b), while Eq. (17) follows from the variation with respect to the cylinder length, which corresponds to a variation of the contour length in method (c).

For the Clifford torus, methods (b) and (c) in Ref. [13] yield a wrong condition on $\vec{P}, \bar{\Sigma}$ and $C_{0}$, since the variations $\delta Z$ for $S=S_{1}$ and $S=S_{2}$ are erroneously taken to be independent. The different results in the two cases arise since there is an additional constraint on the total contour length in method (b).

Thus, we have shown that all problems and inconsistencies mentioned in Ref. [13] result from an improper treatment of the variations at the boundaries. If the variation at the boundaries is carried out correctly, all methods are equivalent and the results do not depend on the method and parametrization chosen.

\section{CONCLUSION}

In conclusion, we have shown that the general shape equation when specialized to an axisymmetric membrane is identical to the shape equation which is obtained if axisymmetry is assumed in the first place. However, the latter method requires the proper inclusion of Lagrange multipliers and the correct treatment of boundary terms. For vesicles of spherical topology a simplification occurs. Once this simplification has been introduced, all subsequent results hold only for spherical topology.
[1] R. Lipowsky, Nature 349, 475 (1991).

[2] M. Wortis, U. Seifert, K. Berndl, B. Fourcade, L. Miao, M. Rao, and R.K.P. Zia, in Proceedings of the Workshop on Dynamical Phenomena at Interfaces, Surfaces and Bilayers, edited by D. Beysens, N. Boccara, and G. Forgacs (Nova, Commack, NY, 1993).

[3] The Structure and Conformation of Amphiphilic Membranes, edited by R. Lipowsky, D. Richter, and K. Kremer (Springer, Berlin, 1992).

[4] W. Helfrich, Z. Naturforsch. C 28, 693 (1973).

[5] H.J. Deuling and W. Helfrich, J. Phys. (Paris) 37, 1335 (1976).

[6] Ou-Yang Zhong-Can and W. Helfrich, Phys. Rev. A 39, 5280 (1989).

[7] We use the convention $H=\left(C_{1}+C_{2}\right) / 2$ where $C_{1}$ and $C_{2}$ are the principal curvatures on the surface. This differs by a factor -1 from the definition for $H$ as used in Ref. [6].
[8] U. Seifert, K. Berndl, and R. Lipowsky, Phys. Rev. A 44, 1182 (1991).

[9] L. Miao, B. Fourcade, M. Rao, M. Wortis, and R.K.P. Zia, Phys. Rev. A 43, 6843 (1991).

[10] S. Svetina and B. Žekš, Eur. Biophys. J. 17, 101 (1989).

[11] U. Seifert, Phys. Rev. Lett. 66, 2404 (1991).

[12] F. Jülicher, U. Seifert, and R. Lipowsky, J. Phys. II 3, 1681 (1993).

[13] Hu Jian-Guo and Ou-Yang Zhong-Can, Phys. Rev. E 47, 461 (1993).

[14] Note that there is one misprint $\left(\sin ^{2} \psi\right.$ instead of $\left.\sin ^{3} \psi\right)$ in Eq. (7) of Ref. [13].

[15] A Clifford torus is parametrized by $R(S)=R_{0}(\sqrt{2}-$ $\cos S)$ and $Z(S)=R_{0} \sin S$.

[16] For an axisymmetric shape with $\mathcal{H} \neq 0$, the second derivative $\ddot{\psi}$ does not vanish at the poles. As a result, the third derivative $\psi^{(3)}$ is discontinuous at the poles. 